\title{
INFLUENCE OF ARTIFICIAL AGEING ON PROPERTIES OF ARCHITECTURAL MEMBRANE AND MEMBRANE CONNECTIONS
}

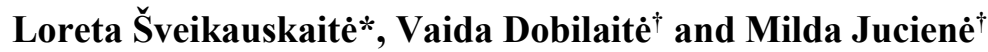 \\ * Kaunas University of Technology, Institute of Architecture and Construction, Tunelio str. 60, \\ 44405 Kaunas, Lithuania \\ E-mail: loreta.sveikauskaite@ktu.edu \\ ${ }^{\dagger}$ Kaunas University of Technology, Institute of Architecture and Construction, Tunelio str. 60, \\ 44405 Kaunas, Lithuania \\ E-mail: vaida.dobilaite@ktu.lt; milda.juciene@ktu.lt
}

\begin{abstract}
Tensioned membrane structures keep popularity due to their engineering benefits and possibilities to implement impressive architectural ideas. However, the expected lifespan of these structures depends on the durability of coated materials used in building and their connections. The performance of both of them leads to a degradation due to exposure to outdoors weathering (high-low temperature, humidity, UV radiation, etc.) and mechanical stresses. In order to insure high level of serviceability the resistance of connections to environmental factors must be known and evaluated in design. This study seeks to investigate the changes in structure and mechanical properties of coated materials and membrane connections exposed to different accelerated aging conditions. PVC-coated polyester fabric suitable for tensioned membrane structure and three types of membrane connections, overlap seam, pocket connection and laced connection (with grommets) were selected for investigation. Hot air welding method was used to produce assembly taken as the research object. For accelerated aging test the specimens including the PES/PVC membrane as well as the connections were divided into two parts. The first part specimens were subjected to hot air at a temperature of $70^{\circ} \mathrm{C}$ and at atmospheric pressure (heat aging). Following the protocol of second part aging the specimens were immersed in water, exposed to cold and exposed to dry heat (cyclic aging). Additionally, before aging the specimens were rubbed with fine (P 180) coated abrasive sheet to damage the protective coating layer in order to simulate an impact of dust and other airborne impurities. The uniaxial tensile and trapezoidal-tear tests were carried out and the characteristics under investigation of the aged PES/PVC membrane and connections from this material with those of the unaged were compared. The failure modes of different membrane connections were also studied to observe the influence of aging factors on membrane / bonding strength. The obtained results showed that in most cases the mechanical properties of membranes and connections change marginally after artificial aging.
\end{abstract}

Keywords: accelerated aging, membranes, membrane connection, tensile strength, tear strength 